\title{
Prisoner's Dilemma and Public Goods Games in Different Geometries: Compulsory Versus Voluntary Interactions
}

\author{
CHRISTOPH HAUERT ${ }^{\mathbf{1}}$ AND GYORGY SZABO ${ }^{2}$ \\ ${ }^{1}$ Department of Zoology, University of British Columbia, 6270 University Boulevard, \\ Vancouver, BC, Canada V6T $1 Z 4$ \\ ${ }^{2}$ Research Institute for Technical Physics and Materials Science, PO Box 49, H-1525 Budapest, Hungary
}

Received August 20, 2002; accepted March 26, 2003

\begin{abstract}
The evolution of cooperation among unrelated individuals in human and animal societies remains a challenging issue across disciplines. In this context, two models have attracted most attention: the prisoner's dilemma for pairwise interactions and the public goods game for group interactions. The two games share many features as demonstrated by the close linkage of their cores. In spatially structured systems with individuals arranged on a lattice we investigate effects of group size and lattice geometry on the success of cooperators and defectors in compulsory and voluntary interactions. The geometry (square versus honeycomb), i.e., the connectivity turns out to have surprisingly pronounced and robust effects on the fate of cooperators. Apparently they thrive more easily on honeycomb lattices. As expected, it becomes increasingly difficult to promote cooperation in sizable groups but voluntary participation significantly lowers the threshold for persistent cooperative behavior. In fact, this effect is even more pronounced for larger groups. The risk avoiding option to not participate provides additional protection to clusters of cooperators against exploitation and introduces rock-scissors-paper-type cyclic dominance, which gives rise to intriguing spatio-temporal patterns. () 2003 Wiley Periodicals, Inc.
\end{abstract}

Key Words: prisoner's dilemma model; public goods game; lattice geometries

\section{INTRODUCTION}

$\mathrm{n}$ biology and social sciences, the prisoner's dilemma (PD) [1] has become the leading paradigm to explain cooperative behavior (see e.g., [2,3]). It captures pairwise interactions where two players have two behavioral options: they decide simultaneously whether to cooperate or to defect. Mutual cooperation pays each player a reward $R$, whereas mutual defection yields the punishment $P$. If a cooperator meets a defector, the former gets the sucker's payoff $S$ and the latter gets away with the temptation to defect $T$. The payoffs must satisfy $T>R>P>S$. Therefore, "rational" players always choose defect because they are better off regardless of the opponent's decision. Thus, ultimately the players end up with the lower punishment $P$ instead of the higher reward $R$-and hence the dilemma. Usually, an altruistic act is characterized by the cost $\gamma$ to the 
donor and the benefit $\beta$ to the recipient. Obviously $\beta>\gamma>$ 0 must hold and we get $R=\beta-\gamma, S=-\gamma, T=\beta$, and $P=0$.

In economics, the related public goods game (PGG) (see e.g., $[4,5])$ attracts increasing attention to study cooperative behavior and, in particular, deviations from the "rational" equilibrium [6]. The PGG describes group interactions where, typically, an experimenter endows, e.g., four players with $\$ 10$ each. The players then have the opportunity to invest part or all of their money into a common pool, knowing that the total amount will be doubled and split equally among all players, irrespective of their contributions. If everybody invests their money, they end up with $\$ 20$. However, each player faces the temptation to free-ride on the other's contributions by withholding the money because each invested dollar yields only a return of 50 cents to the investor. If everybody adopts this "rational" strategy, no one will increase the initial capital and forego the public good.

For both games, traditional and evolutionary game theory invariably predict dominance of asocial, defecting behavior. PD and PGG interactions are abundant in nature. They range from virus strategies, feeding behavior in vampire bats, and predator inspection in sticklebacks to nature conservation, environmental issues, or social security, to name only a few [7-10]. As everybody knows, not all of these examples have evolved into cooperative systems. However, far more important is the surprisingly high and abiding readiness to cooperate. The contrast to theoretical predictions becomes even more striking given the considerable difficulties to establish and maintain cooperative behavior in sizable groups [11].

Advances in theory and experiments have identified several mechanisms capable of promoting and stabilizing cooperation, such as direct or indirect reciprocity [12,13] and reward or punishment [14-17]. In the following, we focus on and combine two further mechanisms: spatial extension [18-20] and voluntary participation $[21,22]$. In spatially extended systems cooperators may thrive by forming clusters, which reduces exploitation through defectors, whereas voluntary participation in public enterprises may provide an escape hatch out of some social traps.

\section{PRISONER'S DILEMMA VERSUS PUBLIC GOODS GAMES}

In order to compare and link the two games, we first generalize the PD to an arbitrary number of players following a simple rule [23]: (a) cooperators obtain $R$ points from every other cooperator and $S$ points from defectors. (b) defectors draw $T$ points from cooperators and $P$ points from other defectors. In groups of $N$ players with $n_{c}$ cooperators (and $N-n_{c}$ defectors) the payoffs are given by

$$
\begin{aligned}
& P_{c}=\left(n_{c}-1\right) R+\left(N-n_{c}\right) S=\left(n_{c}-1\right) \beta-(N-1) \gamma \\
& P_{d}=n_{c} T+\left(N-n_{c}-1\right) P=n_{c} \beta .
\end{aligned}
$$

In the limit of pairwise interactions this reduces to the payoff values introduced above. Also note that with $T>R>$ $P>S$ the dilemma is preserved for any $N$. Regardless of the group composition, defectors are always better off than cooperators, but groups of defectors get only $(N-1) P$ or 0 as opposed to $(N-1) R$ or $(N-1)(\beta-\gamma)$ for mutual cooperation.

Second, we reduce the PGG to its core by considering only two levels of investment: zero, corresponding to defectors withholding their money, or a fixed amount $c$ denoting the cooperators contribution. The value of the public good is determined by the multiplication factor $r$ of the common pool. $r>1$ must hold such that mutual cooperation performs better than mutual defection. Note that for $r>N$ the social dilemma raised by the PGG is relaxed in the sense that each invested dollar has a positive net return. At the same time, the higher gain of defectors is preserved regardless of the group composition. The payoffs for $N$ players engaged in a PGG are then given by

$$
\begin{aligned}
P_{c} & =\left(\frac{r n_{c}}{N}-1\right) c \\
P_{d} & =\frac{r n_{c}}{N} c .
\end{aligned}
$$

From Equations (1), (2) we then obtain the transformation between the PD and PGG:

$$
\begin{aligned}
& \beta \rightarrow \frac{r}{N} c \\
& \gamma \rightarrow \frac{N-r}{N(N-1)} c .
\end{aligned}
$$

Note that the condition $\beta>\gamma$ in the PD becomes equivalent to demanding $r>1$ in the PGG and similarly, from $\gamma>0$ follows $r<N$. Because of this simple transformation, we no longer distinguish between the PD and PGG. Although we generally stick to the PGG, the discussion of various group sizes includes pairwise interactions, i.e., the traditional PD. As an additional simplification but without loss of generality, we normalize the investment costs $c$ of the PGG to unity.

\section{VOLUNTEERS IN SPACE}

So far we implicitly assumed compulsory participation in the PGG by restricting the behavioral options to cooperation and defection. In nature, however, individuals often have the ability and possibility to choose their partners or to abstain from unpromising public enterprises. We model this 


\section{FIGURE 1}
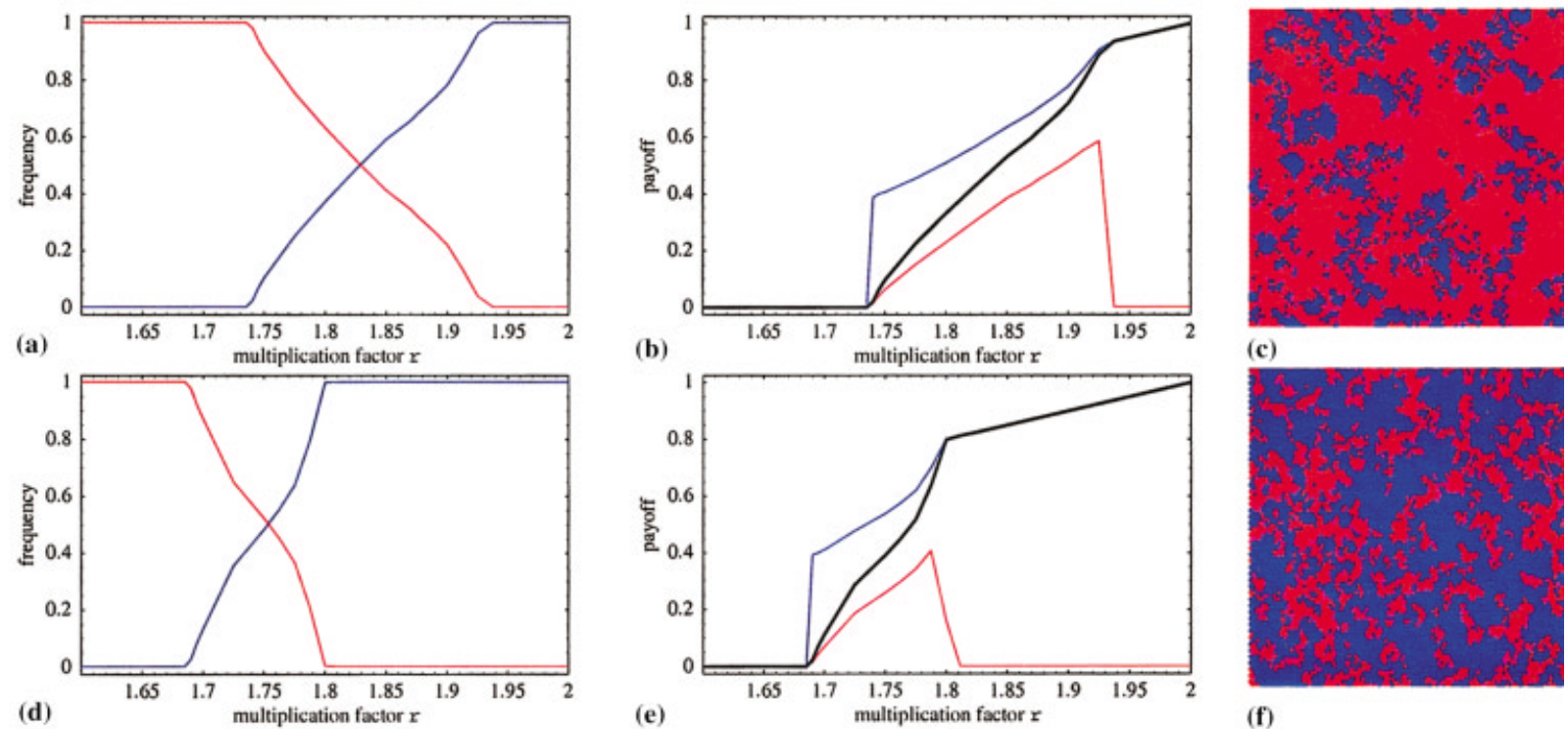

(c)

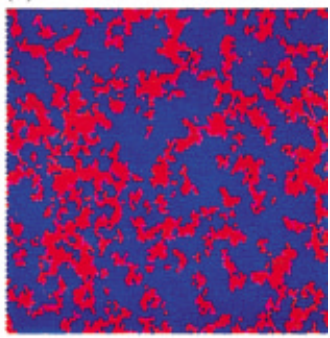

(f)

Pairwise interactions in the compulsory PGG on square (a)-(c) versus honeycomb lattices (d)-(f): equilibrium frequencies $(a, d)$ and payoffs $(b, e)$ of cooperators (blue) and defectors (red) as a function of the multiplication factor $r$ together with typical snapshots of the lattice for $r=1.78$ (c,f). The bold black line shows the average population payoff $\bar{P}$. Below the threshold value $r<r_{c}$ cooperators quickly vanish, whereas for high $r>r_{d}$ defectors go extinct. For intermediate $r$ the two strategies co-exist in dynamical equilibrium. As soon as cooperators are able to survive $\left(r>r_{c}\right)$, their average payoff is consistently and significantly higher than for defectors. Interestingly, honeycomb lattices promote cooperation as indicated by the lower threshold value $r_{c} \approx 1.675$, contrasting with $r_{c} \approx 1.735$ on a square lattice. Similarly, cooperators are capable of displacing defectors already for significantly lower $r$. A $200 \times 200$ lattice with periodic boundaries is randomly initialized with $80 \%$ cooperators and $20 \%$ defectors. This asymmetry compensates for the inherent advantage of defectors in random environments and reduces the risk of accidental extinctions of cooperators due to random fluctuations. The system is then relaxed over 5000 Monte-Carlo (MC) steps. One such step covers $M$ interactions where $M$ stands for the number of sites on the lattice. The equilibrium frequencies and payoffs are determined by averaging over another $5000 \mathrm{MC}$ steps. To improve the accuracy in the vicinity of $r_{c}$ and $r_{d}$, several simulations were run on $300 \times 300$ lattices and relaxation times of $10^{4} \mathrm{MC}$ steps. The snapshots show a $100 \times 100$ lattice after $300 \mathrm{MC}$ steps where intermediate color shades indicate sites that have recently changed their strategy.

by introducing a third strategical character: the loners. Loners are risk averse and refuse to participate in PGGs. They prefer an autarkic way of living and rather rely on a small but fixed source of income $\sigma$ with $0<\sigma<r-1$. The two inequalities indicate that loners are assumed to perform better than groups of defectors but worse than mutual cooperation. Because the payoffs now depend on the number of loners in the group, we need to modify Equation (2):

$$
\begin{aligned}
P_{c} & =\frac{r n_{c}}{n_{c}+n_{d}}-1 \\
P_{d} & =\frac{r n_{c}}{n_{c}+n_{d}} \\
P_{l} & =\sigma,
\end{aligned}
$$

where $n_{c}+n_{d}$ indicates the actual number of participants in the PGG. Solitary cooperators or defectors are assumed to act as loners. The three strategies exhibit cyclic dominance: if cooperators abound, it pays to defect, but if defectors prevail, it is best to abstain, and if no one participates, small groups can form which render cooperation attractive again. Although voluntary participation is not able to stabilize cooperation, it provides a natural way to avoid deadlocks in states of mutual defection and economic stalemate. It enables cooperators to thrive and persist at substantial levels even in sizable groups, in absence of repeated interactions and under full anonymity [21]. In well-mixed populations with replicator dynamics [24] this system can be solved analytically despite the highly nonlinear payoff terms [22].

In this article, we consider spatially extended variants of the PGG where players are arranged on a rigid regular lattice and interact only within their immediate neighborhood. The size of the neighborhood determines the maximum number of participants $N$ in the PGG. In the following we discuss various different neighborhood types on square and honeycomb lattices ranging from pairwise interactions to the Moore neighborhood with $N=9$. According to the principles of evolutionary game theory [25], we assume that the average payoff achieved in PGG interactions, i.e., the 

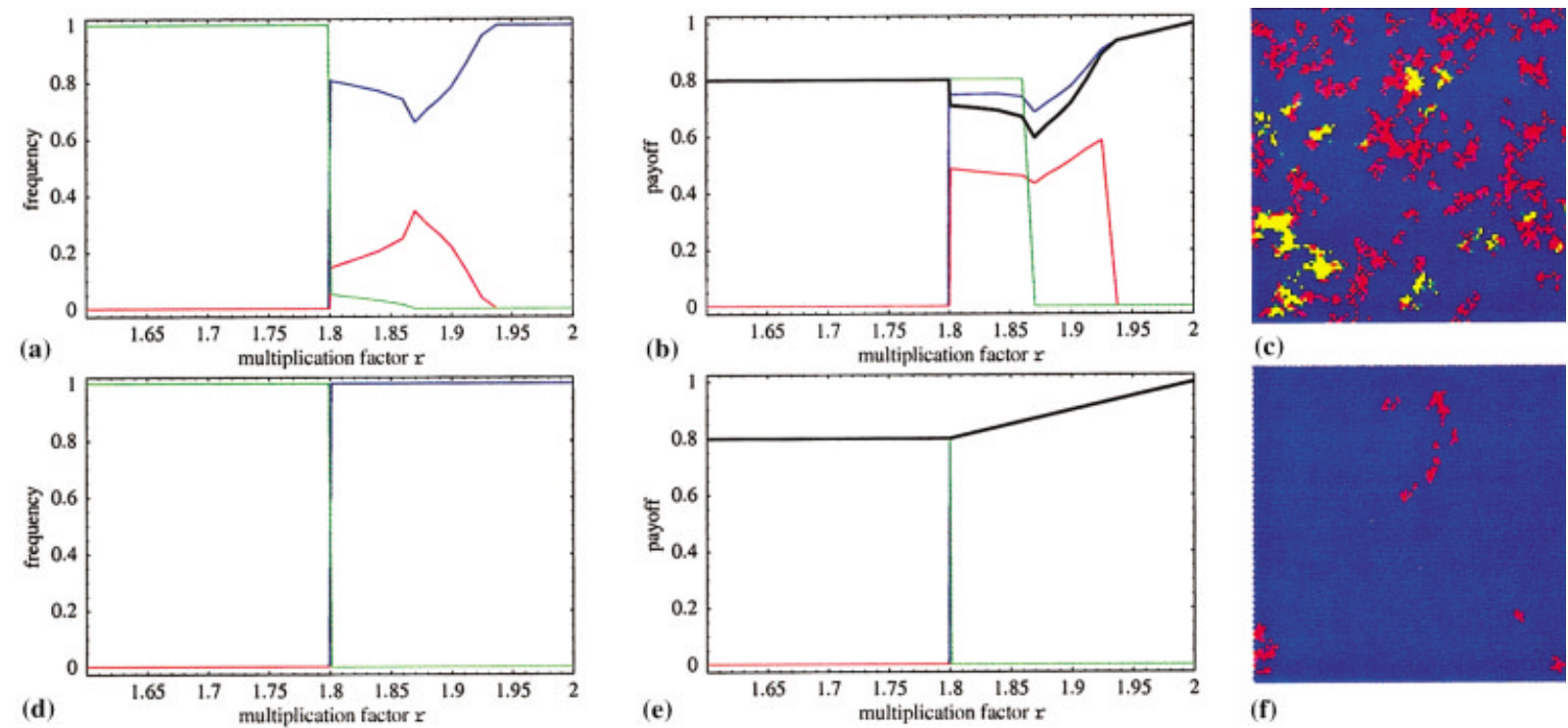

(f)

Pairwise interactions in the voluntary PGG on square $(a-c)$ versus honeycomb lattices $(d-f)$ : equilibrium frequencies $(a, d)$ and payoffs $(b, e)$ of cooperators (blue), defectors (red), and loners (green) as a function of $r$ with $\sigma=0.8$ together with typical snapshots for $r=1.82$ (loners yellow). The bold black line indicates the average population payoff $\bar{P}$. For $r<1+\sigma$ loners dominate because they outperform even groups of cooperators. Above this threshold cooperators immediately take over on honeycomb lattices, whereas on square lattices a domain of co-existence follows. At first, all three strategies co-exist until for $r>r_{1} \approx 1.86$ loners go extinct and cooperators thrive without their assistance. Soon after, for $r>r_{d} \approx 1.925$, defectors follow and only cooperators remain. In (f) the system has not yet completely relaxed and few small patches of defectors still exist, bound to vanish soon. The simulation parameters are the same as specified in Figure 1, except that at initialization the frequencies of the strategies were set to $20 \%$ defectors with $40 \%$ cooperators and loners.

score of a player determines its reproductive success. In the present context this refers to the probability that neighbors imitate and adopt the player's strategy $[24,26]$.

In order to determine the equilibrium frequencies of the strategies, the lattice is evolved in time according to the following procedure: a randomly selected player compares his score to those of his neighbors and switches to better performing strategies with a probability proportional to the difference in scores but sticks to his current strategy otherwise. Note that this represents a spatial analogue of the replicator or imitation dynamics. Whenever a player decides to switch strategy, its score is reset. After reconsidering the strategy, a PGG is offered to the player and its neighbors. Finally, the scores of all players involved are updated according to their individual performance.

\section{PAIRWISE INTERACTIONS}

Pairwise interactions, i.e., the PD, in spatially extended systems and the emergent complex dynamics have been extensively studied in the literature [27-29]. The spatial extension enables cooperators to prosper through cluster formation and thereby reducing exploitation by defectors. In well-mixed populations with random encounters and identical game parameters, cooperators do not stand a chance and invariably defectors dominate.

Here we focus on two other aspects: effects of the lattice geometry as well as voluntary participation on the equilibrium frequency of the strategies. Let us first consider the compulsory game on the traditional square lattice compared to honeycomb or hexagonal lattices. On the square lattice a randomly selected player compares its score to a randomly chosen neighbor out of a Moore neighborhood, i.e., out of the eight adjacent sites reachable by a chesskings-move. Similarly, on a honeycomb lattice, one neighbor is chosen at random out of the six adjoining sites. After reassessing its strategy, the player interacts with possibly another, again randomly chosen, neighbor out of the same neighborhood. Figure 1 shows the equilibrium frequencies and payoffs of cooperators and defectors as a function of the multiplication factor $r$. On both lattice types three dynamical domains are identified: for low $r<r_{c}$ cooperators are doomed and defectors dominate, whereas for sufficiently high $r>r_{d}$ the clustering advantage of cooperators becomes large enough to eventually displace all defectors. Intermediate values of $r_{c}<r<r_{d}$ lead to co-existence of the two strategies. The index $\alpha$ of the thresholds $r_{\alpha}$ always refers 


\section{FIGURE 3}

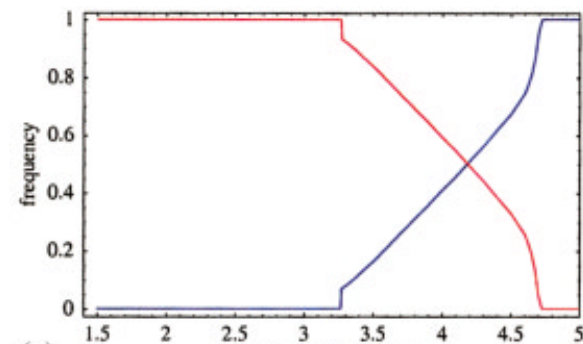

(a)

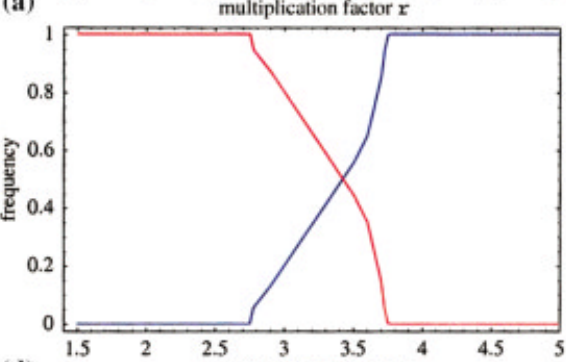

(d)

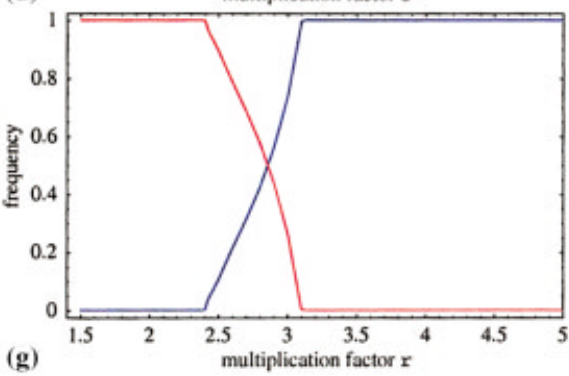

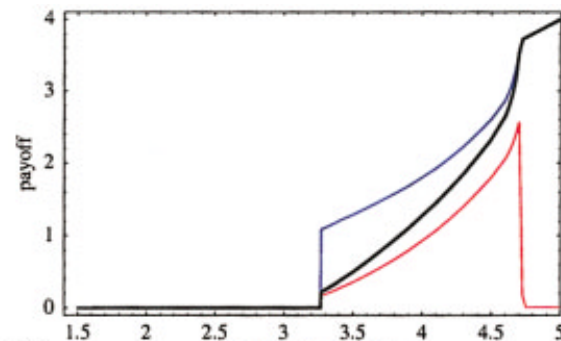

(b)

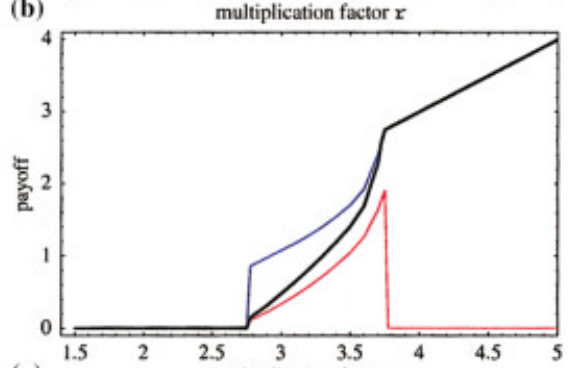

(e)

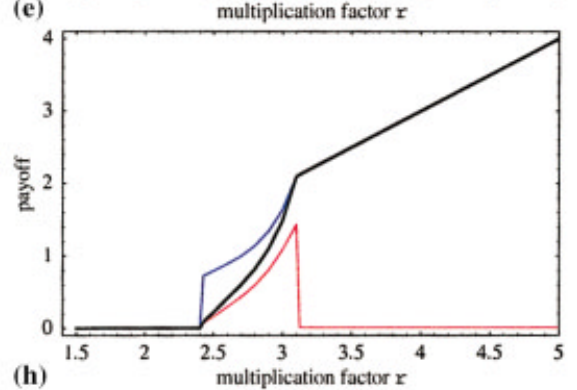

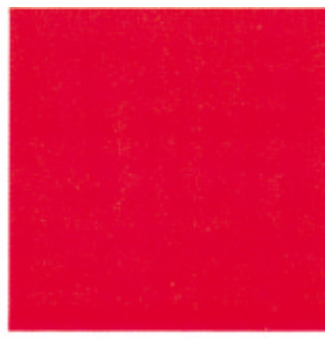

(c)

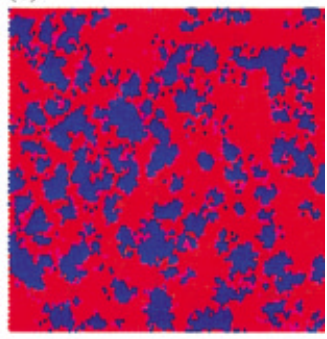

(f)

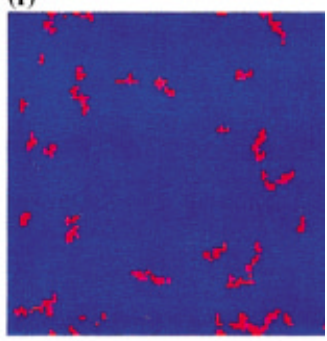

(i)

Equilibrium frequencies $(\mathrm{a}, \mathrm{d}, \mathrm{g})$ and payoffs $(\mathrm{b}, \mathrm{e}, \mathrm{h})$ of cooperators (blue) and defectors (red) on regular lattices in the compulsory PGG as a function of $r$ together with typical snapshots for $r=3.2$ (c,f,i). The bold black line indicates the average population payoff $\bar{P}$. The group size $N$ decreases from top to bottom: $(\mathrm{a}-\mathrm{c})$ square lattice with Moore neighborhood $N=9$, (d-f) honeycomb lattice $(N=7)$ and $(g-l)$ square lattice with the smaller von Neumann neighborhood $(N=5)$. The dynamics displays three domains (cf., Figure 1): below the threshold $r_{c}$ cooperators quickly vanish, but for $r>r_{d}$ they eventually displace all defectors and for intermediate values $r_{c}<r<r_{d}$ the two strategies co-exist. The group size $N$ dominantly determines the fate of cooperators: first, the threshold for persisting cooperation $r_{c}$ and second, the region of co-existence both increase with $N$ (see Table 1). Both effects underline the increased difficulty to establish cooperation in sizable groups. All simulation parameters are as specified in Figure 1.

to the vanishing strategy. Despite these identical qualitative features, surprisingly pronounced differences are observed on the quantitative level originating solely in the different geometries. Regardless of considerable noise in the update procedure, it is apparently far easier for cooperators to prosper on honeycomb lattices, as reflected by the significantly lower threshold values $r_{c}, r_{d}$ for the appearance/ extinction of cooperators and defectors, respectively. The reason for this difference lies in the connectivity of the two lattice types, i.e., in the different numbers of potential partners. With a higher individual connectivity, clusters of cooperators are more exposed to exploitation. Consequentially it becomes more difficult to establish cooperation on a square lattice with the Moore neighborhood (eight neighbors) than on a honeycomb lattice with six neighbors.

\section{TABLE 1}

Approximate Threshold Values $r_{c}, r_{d}$ and Width $r_{c}-r_{d}$ of the Co-existence Region for All Neighborhood Types under Consideration

\begin{tabular}{lllll}
\hline$N$ & \multicolumn{1}{c}{$r_{c}$} & \multicolumn{1}{c}{$r_{d}$} & Width & \multicolumn{1}{c}{ Neighborhood } \\
\hline 2 & 1.675 & 1.8 & 0.125 & Pairwise (honeycomb) \\
2 & 1.735 & 1.9375 & 0.2025 & Pairwise (square) \\
5 & 2.4 & 3.1 & 0.7 & von Neumann \\
7 & 2.75 & 3.75 & 1.0 & Honeycomb \\
9 & 3.265 & 4.725 & 1.46 & Moore \\
\hline
\end{tabular}

All values increase with group size $N$ confirming that it gets increasingly difficult to promote cooperation in larger groups, i.e., requiring significantly higher $r$ and widening the region of co-existence. 


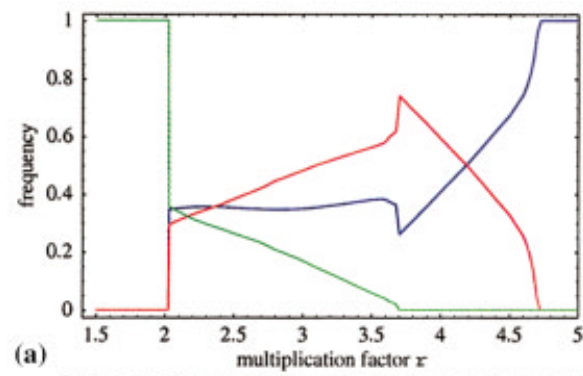

(a)

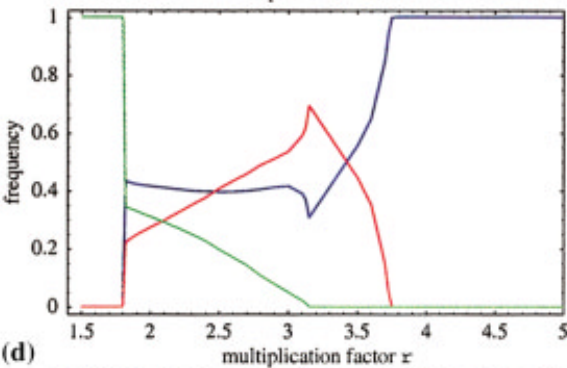

(d)

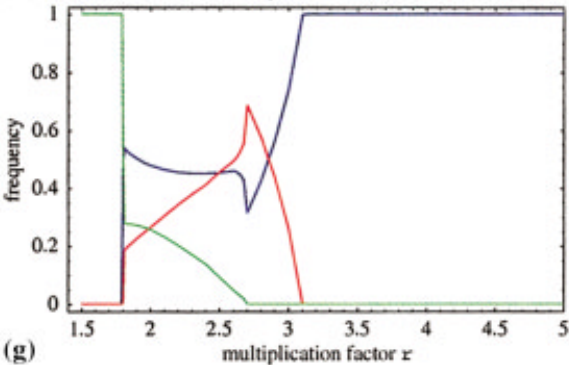

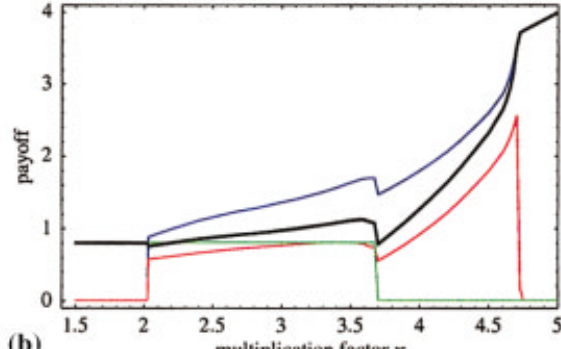

(b)

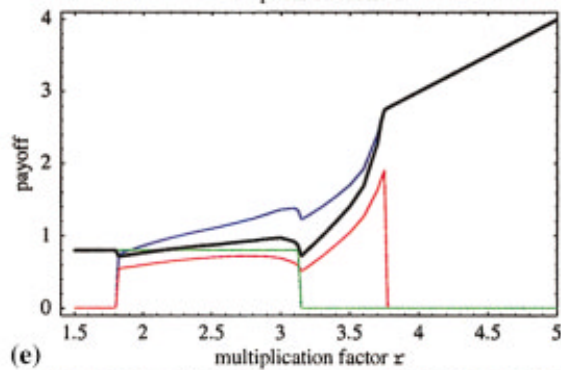

(e)

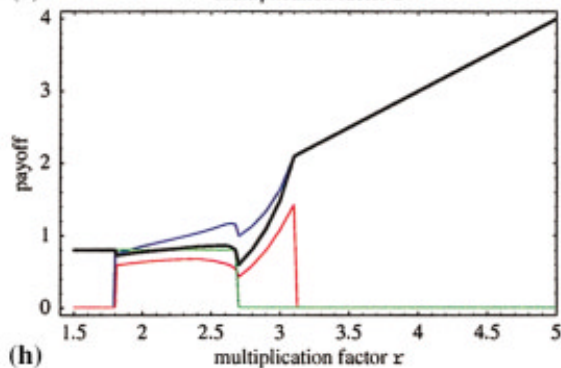

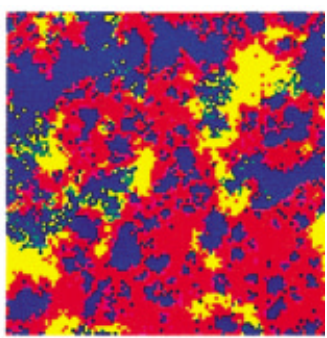

(c)

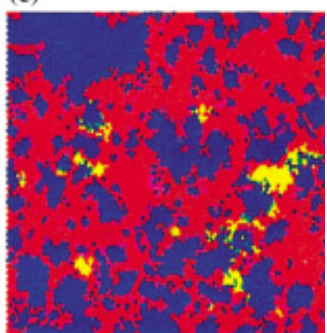

(f)

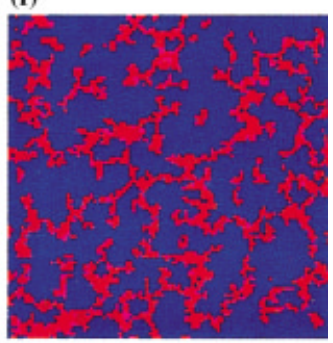

(i)

Equilibrium frequencies (a,d,g) and payoffs (b,c,h) of cooperators (blue), defectors (red), and loners (green) in voluntary PGG as a function of $r$ with $\sigma=$ 0.8 , together with typical snapshots for $r=3.0$ in (c,f,i) (loners yellow). The bold black line indicates the average population payoff $\bar{P}$. The graphs are arranged as in Figure 3: (a-c) Moore neighborhood $(N=9)$, $(\mathrm{d}-\mathrm{f})$ honeycomb lattice $(N=7)$, $(\mathrm{g}-\mathrm{i})$ von Neumann neighborhood $(N=5)$. For $r<1+\sigma$ loners dominate but above that threshold or soon after, a domain follows with all three strategies co-existing. For larger $r>r_{b}$, cooperators thrive on their own and loners go extinct. Consequentially, the results become identical to the compulsory game (cf., Figure 3). Voluntary participation significantly increases the parameter range where cooperative behavior persists (see Table 2), an effect that is even more pronounced for larger group sizes.

Allowing for voluntary participation, i.e., introducing the loner strategy, considerably affects the fate of cooperators and defectors. The equilibrium frequencies and payoffs are shown in Figure 2 for a fixed income of loners with $\sigma=0.8$. For $r<1+\sigma$ loners trivially dominate because they gain even more than groups of cooperators. On honeycomb lattices the regions of co-existence are missing. Once mutual cooperation outperforms loners $(r>1+\sigma)$, cooperators readily and seamlessly take over. This is not too surprising because in absence of loners, cooperators took over for $r_{d}<$ $1+\sigma$. In contrast, on square lattices [see Figure 2(a,b)] above this threshold all three strategies co-exist giving rise to intriguing spatio-temporal patterns. Somewhat surprisingly this is accompanied by a drop in average population payoff $\bar{P}<\sigma$. This indicates that the population would be better off without the opportunity to participate in the PGG, i.e., in homogenous states of all loners. Above another threshold $r_{l} \approx 1.86$ loners no longer provide a viable option and vanish. One might say that the players have, out of free will, changed the voluntary game into a compulsory one. In this domain $\bar{P}$ quickly recovers and exceeds $\sigma$. For $r>r_{l}$ cooperators thrive on their own and soon displace defectors entirely (cf., Figure 1).

Interestingly, in well-mixed populations the voluntary PGG with pairwise interactions $(r<2)$ always relaxes into a homogenous state of all loners. Only in spatially structured systems, the loner strategy becomes a valuable alternative providing additional protection to cooperative clusters against exploitation by defectors. However, this mechanism becomes apparent only for smaller values of $\sigma$, i.e., for $\sigma<$ 
$r_{c}-1$. In order to simplify comparisons we kept $\sigma$ fixed for all simulations presented here. This results in fairly large values for pairwise interactions, but $\sigma$ appears far smaller in groups as large as $N=9$, which generally require higher $r$.

\section{GROUP INTERACTIONS}

According to the original application of the PGG we now turn to larger groups of interacting individuals. On square lattices we consider the Moore neighborhood with $N=9$ consisting of the chosen player and its eight nearest neighbors and the smaller von Neumann neighborhood including only the four neighbors to the north, east, south, and west resulting in $N=5$. An intermediate size with $N=7$ is considered on a honeycomb lattice.

For all three neighborhood sizes the equilibrium frequencies of cooperators and defectors and their payoffs are shown in Figure 3 for the compulsory game, i.e., in absence of the loner strategy. The qualitative features discussed for pairwise interactions remain unchanged for larger $N$. Again three domains are observed: for low $r<r_{c}$ defectors dominate, co-existence for intermediate values and homogenous cooperation for high $r>r_{d}$. Once cooperators persist, they achieve significantly higher payoffs than defectors.

On quantitative levels, interesting differences and trends are observed (see Table 1). The threshold value $r_{c}$ as well as the width of the region of co-existence increases with $N$. Both trends indicate that it becomes increasingly difficult to establish cooperation in larger groups.

Voluntary participation has pronounced effects on the fate of cooperators in larger groups (see Figure 4). In fact, the simulations suggest that the cooperators advantage tends to increase with $N$. For $r<1+\sigma$ loners invariably dominate. Above this threshold or soon after, a region follows where all strategies co-exist in dynamical equilibrium. The rock-scissors-paper-type dominance of cooperators, defectors and loners induces traveling waves sweeping across the lattice and give rise to intriguing dynamically changing patterns. Increasing $r$ further eventually leads to the extinction of loners at $r_{l}$. For $r>r_{l}$ the system is equivalent to the compulsory game (see Figure 3 ) because loners no longer provide a viable alternative.

The increase in the parameter range of persistent cooperative behavior is shown in Table 2 for all $N$ under consideration. The threshold $r_{c}$ is shifted toward significantly lower $r$. This results from the additional protection provided by loners: obviously they mitigate exploitation simply by occupying sites along the cluster boundary, but in addition, they reduce the number of participants in the PGG, which may result in groups small enough to relax the social dilemma and render cooperation more attractive [22]. Note that in the voluntary PGG both cooperators and defectors vanish at $r_{c}$ leaving the field to loners. For a detailed discussion of second-order phase transitions, occurring when co-

\section{TABLE 2}

Approximate Threshold Values $r_{c}, r_{l}$ and $r_{d}$ (note, at $r_{c}$ both cooperators and defectors vanish)

\begin{tabular}{llllcl}
$N$ & $r_{c}$ & \multicolumn{1}{c}{$r_{l}$} & \multicolumn{1}{c}{$r_{d}$} & Advantage & \multicolumn{1}{c}{ Neighborhood } \\
\hline 2 & 1.8 & 1.8 & 1.8 & - & Pairwise (honeycomb) \\
2 & 1.8 & 1.87 & 1.9375 & - & Pairwise (square) \\
5 & 1.8 & 2.7 & 3.1 & 0.6 & von Neumann \\
7 & 1.8 & 3.15 & 3.75 & 1.35 & Honeycomb \\
9 & 2.03 & 3.7 & 4.725 & 1.235 & Moore \\
\hline
\end{tabular}

For $r_{c}<r<r_{\text {l }}$ all three strategies co-exist, but for $r>r_{\text {l }}$ loners go extinct leaving a mixture of cooperators and defectors behind $\left(r_{1}<\right.$ $r<r_{d}$ ). The advantage indicates the shift of $r_{c}$ due to voluntary participation compared to the compulsory PGG. For $\sigma=0.8$ the loner option did not increase the cooperative range in pairwise interactions because of $r_{c}<1+\sigma$. Apparently the advantage provided by voluntary participation tends to increase with $N$, but the honeycomb lattice seems to further promote cooperation such that the biggest advantage is obtained for $N=7$.

operators go extinct for $r \rightarrow r_{c}$ in the compulsory game and in the voluntary variant for the extinction of defectors for $r$ $\rightarrow 1+\sigma$, as well as their relation to direct percolation, we refer to Szabó and Hauert [30].

To illustrate the potential complexity of the emerging spatio-temporal patterns, we note that in principle, the spatial PGG can be formulated as a probabilistic cellular automaton [31], but for the Moore neighborhood this requires a noticeable number of $3^{49} \approx 10^{23}$ transition rules. The dynamics of this neat system can be interactively verified and further explored at Hauert's Web site [32]. Note that for $r$ in the region where all three strategies co-exist together with synchronized lattice updates and deterministic update rules for the players, i.e., imitate the best neighbor (including himself) with certainty, fascinating evolutionary kaleidoscopes are obtained for symmetrical initial conditions. Admittedly, this is of limited scientific interest but deserves attention for its entertainment value.

Interestingly, for $N>2$ the average population payoff $\bar{P}$ remains within narrow limits and very close to $\sigma$ for $r$ in the region of co-existence including all three strategies. This is related to findings for well-mixed populations, where voluntary participation induces a red queen mechanism, which results in an equal long-term average payoff $\sigma$ for all strategies. Therefore, everybody would do equally well without the possibility to participate in a PGG. However, at the same time, note that everybody is significantly better off in voluntary PGG interactions than in compulsory games.

For pairwise interactions, cooperation persists only in spatially extended systems [33]. The compulsory PGG leads to economic stalemate in states of mutual defection, whereas in 
the voluntary game no one is willing to participate, and all opt for the loner strategy. In both cases cluster formation enables cooperators to prosper, but voluntary participation may further increase the range of suitable $r$ for appropriately chosen $\sigma$.

Changing compulsory into voluntary participation by introducing the risk averse loners results in a rock-scissorspaper type dominance of the three strategies. In spatial settings this results in intriguing spatio-temporal patterns [34-36]. Most importantly, however, it enables cooperators to thrive for a much wider parameter range. In particular, cooperative behavior persists already for significantly lower multiplication factors $r$, i.e., smaller values of the public good. The group size predominantly determines the fate of cooperators mainly characterized by the thresholds for persistent cooperative behavior. Cooperation in larger groups requires significantly higher $r$ in compulsory games, whereas with voluntary participation this effect is largely neutralized. In fact, effects of the loner option are even more pronounced in larger groups. In addition, we demonstrated that the lattice geometry, i.e., the connectivity or the number of potential partners has additional effects: for pairwise interactions on honeycomb lattices with six neighbors cooperation spreads more easily than on square lattices with eight neighbors.

\section{REFERENCES}

1. Axelrod, R.; Hamilton, W.D. The evolution of cooperation. Science 1981, 211, 1390.

2. Colman, A.M. Game Theory and its Applications in the Social and Biological Sciences; Butterworth-Heinemann: 0xford, 1995.

3. Dugatkin, L.A. Cooperation among Animals: An Evolutionary Perspective. Oxford University Press: Oxford, 1997.

4. Kagel, J.H.; Roth, A.E. The Handbook of Experimental Economics; Princeton University Press: Princeton, 1995.

5. Binmore, K.G. Fun and Games: A Text on Game Theory; Heath \& Co: Lexington, MA, 1992.

6. Fehr, E.; Gächter, S. Altruistic punishment in humans. Nature 2002, 415, 137.

7. Turner, P.E.; Chao, L. Prisoner's dilemma in an RNA virus. Nature 1999, 398, 441.

8. Wilkinson, G.S. Reciprocal food-sharing in the vampire bat. Nature 1984, 308, 181.

9. Milinski, M. Tit for tat in sticklebacks and the evolution of cooperation. Nature 1987, 325, 433.

10. Binmore, K.G. Playing Fair: Game Theory and the Social Contract. MIT Press: Cambridge, 1994.

11. Boyd, R.; Richerson, P.J. The evolution of reciprocity in sizeable groups. J Theor Biol 1988, 132, 337.

12. Trivers, R.L. The evolution of reciprocal altruism. Q Rev Biol 1971, 46, 35.

13. Nowak, M.A.; Sigmund, K. Evolution of indirect reciprocity by image scoring. Nature 1998, 393, 573.

14. Fehr, E.; Gächter, S. Cooperation and punishment in public goods experiments. Am Econ Rev 2000, 90, 980.

15. Boyd, R.; Richerson, P.J. Punishment allows the evolution of cooperation (or anything else) in sizable groups. Ethology Sociobiol 1992, $13,171$.

16. Milinski, M.; Semmann, D.; Krambeck, H.-J. Reputation helps solve the 'tragedy of the commons'. Nature 2002, 415, 424.

17. Sigmund, K.; Hauert, C.; Nowak, M.A. Reward and punishment. Proc Natl Acad Sci USA 2001, 98, 10757.

18. Nowak, M.A.; May, R.M. Evolutionary games and spatial chaos. Nature 1992, 359, 826.

19. Szabó, G.; Tóke, C. Evolutionary prisoner's dilemma game on a square lattice. Phys Rev E 1998, 58, 69.

20. Hauert, C. Effects of space in $2 \times 2$ games. Int J Bifurcation Chaos 2002, 12, 1531.

21. Hauert, C.; De Monte, S.; Hofbauer, J.; Sigmund, K. Volunteering as red queen mechanism for cooperation in public goods games. Science 2002, $296,1129$.

22. Hauert, C.; De Monte, S.; Hofbauer, J.; Sigmund, K. Replicator dynamics in optional public goods games. J Theor Biol 2002, 218, 187.

23. Dawes, R.M. Social dilemmas. Annu Rev Psychol 1980, 31, 169.

24. Hofbauer, J.; Sigmund, K. Evolutionary Games and Population Dynamics; Cambridge University Press: Cambridge, 1998.

25. Maynard Smith, J. Evolution and the Theory of Games; Cambridge University Press: Cambridge, 1982.

26. Schlag, K. Why imitate, and if so, how? A bounded rational approach to multi-armed bandits. J Econ Theor 1998, 78, 130.

27. Killingback, T.; Doebeli, M. Self-organized criticality in spatial evolutionary game theory. J Theor Biol 1998, 191, 335.

28. Hubermann, B.A.; Glance, N.S. Evolutionary games and computer simulations. Proc Natl Acad Sci USA 1993, 90, 7712.

29. Nowak, M.A.; Bonhoeffer, S.; May, M. Spatial games and the maintenance of cooperation. Proc Natl Acad Sci USA 1994, 91, 4877.

30. Szabó, G.; Hauert, C. Phase transitions and volunteering in spatial public goods games. Phys Rev Lett 2002, 89, 118101.

31. Wolfram, S. Theory and Applications of Cellular Automata; World Scientific: Singapore, 1986.

32. Hauert, C. Virtual labs for volunteering in public goods games. URL http://www.univie.ac.at/virtuallabs, 2002.

33. Szabó, G.; Hauert, C. Evolutionary prisoner's dilemma with optional participation. Phys Rev E 2002, 66, 062903.

34. Frean, M.; Abraham, E.R. Rock-scissors-paper and the survival of the weakest. Proc R Soc Lond B 2001, 268, 1323.

35. Tainaka, K. Vortices and strings in a model ecosystem. Phys Rev E 1994, 50, 3401.

36. Szabó, G.; Antal, T.; Szabó, P.; Droz, M. Spatial evolutionary prisoner's dilemma game with three strategies and external constraints. Phys Rev E 2000, 62, 1095. 